\title{
5.1 The Role of Blood Pressure, Aortic Stiffness, and Haemodynamics in Brain Health in Older People with Type 2 Diabetes Mellitus
}

\author{
Christopher Karayiannis ${ }^{1, *}$, Chris Moran ${ }^{1}$, Richard Beare ${ }^{1}$, James Sharman ${ }^{2}$, Thanh Phan ${ }^{3}$, Amanda Thrift ${ }^{3}$, \\ Wei Wang ${ }^{1}$, Velandai Srikanth ${ }^{1}$
}

${ }^{1}$ Peninsula Health, Melbourne, Australia

${ }^{2}$ University of Tasmania, Tasmania, Australia

${ }^{3}$ Monash University, Melbourne, Australia

\section{ABSTRACT}

Aims: Type 2 diabetes (T2D) is associated with dementia, and abnormal blood pressure (BP), aortic stiffness, and haemodynamics. We aimed to study the contribution of such factors to brain structure and cognition, relative to the contribution of metabolic factors in T2D such as insulin resistance and adiposity, in older people with T2D.

Methods: Cross-sectional sample with T2D in the Cognition and Diabetes in Older Tasmanians-Blood Pressure (CDOT-BP) study. Measurements included neuropsychological battery, brain MRI, and Mobil-o-Graph-derived (IEM, Germany) aortic BP and haemodynamics. We studied associations of exposure variables with outcomes using structural equation modelling for cognition, and simple linear regression for brain structure, using standardised coefficients to determine relative contribution of variables to the outcomes in combined regression models.

Results: 202 participants (mean age 68.5 years, mean $\mathrm{HbAlc}=6.42 \%$ ). Augmentation index was associated with processing speed $(p<0.05)$. The contribution of augmentation index to processing speed (standardized $\beta=-0.237$ ) was greater than waisthip ratio $(\beta=-0.175)$ and use of oral glucose lowering medications and insulin $(\beta=-0.126)$, but less than age $(\beta=-0.431)$. Peripheral systolic BP, pulse wave velocity, and peripheral nocturnal systolic BP dipping were associated with measures of white matter integrity (fractional anisotropy and mean diffusivity, $p<0.05$ ) but not with cortical thickness.

Conclusion: BP, aortic stiffness and altered haemodynamics contribute to early cognitive change and loss of white matter integrity in T2D. The novel association between nocturnal BP dipping and white matter integrity deserves further investigation. Measuring white matter integrity may be useful to detect the effect of these vascular factors on the brain.

(c) 2019 Association for Research into Arterial Structure and Physiology. Publishing services by Atlantis Press International B.V. This is an open access article distributed under the CC BY-NC 4.0 license (http://creativecommons.org/licenses/by-nc/4.0/). 